\title{
Formation and Mechanical Properties of Pd-Si Binary Bulk Metallic Glasses
}

\author{
Na Chen, Hongxia Zhang, and Ke-Fu Yao \\ School of Materials Science and Engineering, Tsinghua University, Beijing 100084, China \\ Correspondence should be addressed to Na Chen; chennadm@mail.tsinghua.edu.cn and Ke-Fu Yao; kfyao@tsinghua.edu.cn
}

Received 17 March 2014; Accepted 7 May 2014; Published 28 May 2014

Academic Editor: Yang Shao

Copyright (C) $2014 \mathrm{Na}$ Chen et al. This is an open access article distributed under the Creative Commons Attribution License, which permits unrestricted use, distribution, and reproduction in any medium, provided the original work is properly cited.

Glassy spherical samples in the diameters up to $10 \mathrm{~mm}$ were produced in a binary Pd-Si alloy system. These Pd-Si bulk metallic glasses (BMGs) combine high strength of about $1600 \mathrm{MPa}$ and superplasticity of over $70 \%$ together. In addition to abundant micrometer-scale shear bands, 10-20 nanometer-sized shear bands were also observed on the side surface of the deformed sample. The excellent ductility shown by the Pd-Si BMGs is suggested to arise from the nanoscale structural inhomogeneity.

\section{Introduction}

The formation of the first metallic glass (MG) in a binary AuSi system has spurred intensive interest in the fundamental understanding of glass formation and structure-property relationship [1-5]. In particular, the unique mechanical response of bulk metallic glasses (BMGs) makes them more attractive in certain application fields than their crystalline counterparts [6-10]. Based on a "confusion principle," BMGs are usually formed in multicomponent alloy systems [2-4]. However, some binary alloy systems such as $\mathrm{Cu}-\mathrm{Zr}$, Pd-Si, and $\mathrm{Cu}-\mathrm{Hf}$ as well as $\mathrm{Ni}-\mathrm{Nb}$ alloys exhibit exceptionally high glass-forming ability (GFA) so that BMGs can be produced from these alloys [11-15]. In addition, these binary BMGs show a combination of both high strength and large ductility $[8,9]$. Due to their simple compositions, the binary alloys are more prone to crystallization than the multicomponent alloys, posing extreme difficulties in producing BMGs with sizes larger than $2 \mathrm{~mm}$ [11-14]. In this paper, Pd-Si BMGs in the diameter up to $10 \mathrm{~mm}$ were produced, which are the largest binary BMGs reported so far. On the basis of the nanoindentation and uniaxial compression tests, the mechanical properties of $\mathrm{Pd}_{81} \mathrm{Si}_{19} \mathrm{BMG}$ alloys have been studied.

\section{Experimental Procedure}

Ingots of $\mathrm{Pd}_{81} \mathrm{Si}_{19}$ alloys were prepared by melting the mixtures of Pd and Si with over 99.99 wt\% purities in an argon atmosphere. $\mathrm{Pd}_{81} \mathrm{Si}_{19}$ ingots were continuously purified by the fluxing medium including $\mathrm{B}_{2} \mathrm{O}_{3}$ in a quartz tube located in a stove with a constant temperature of $1423 \mathrm{~K}$ till glassy alloys were obtained. During this process, the quartz tube was taken out of the stove and cooled under air every 30 minutes in order to promote the fluxing effect. The structure of the glassy alloys was examined by X-ray diffractometry (XRD) with monochromatic $\mathrm{CuK}_{\alpha}$ radiation. The elastic moduli of the $\mathrm{Pd}_{81} \mathrm{Si}_{19}$ BMGs, such as Young's modulus and the shear modulus, were obtained by the ultrasonic measurements. The microstructure of the samples was observed by transmission electron microscope (TEM) using a JEM 120EX (JEOL) and a JEM 2010F high resolution transmission electron microscope (HRTEM) with an accelerating voltage of $200 \mathrm{KV}$. The thin foil specimens for TEM observation were prepared by a standard twin-jet electropolishing method. Nanoindentation tests were performed at a strain rate of $0.5 \mathrm{~s}^{-1}$ by the Nano Indenter indentation instrument of MTS with Berkovich tip. Uniaxial compression tests were measured by an Instron testing machine.

\section{Results and Discussions}

Figure 1 shows the XRD patterns of Pd-Si binary alloys. The diameter of the spherical $\mathrm{Pd}_{81} \mathrm{Si}_{19}$ sample is about $10 \mathrm{~mm}$ while the diameter of the $\mathrm{Pd}_{80} \mathrm{Si}_{20}$ glassy ball is around $8 \mathrm{~mm}$. Except the broad diffraction peaks, no distinctive sharp diffraction peak resulted by crystalline phases can be 


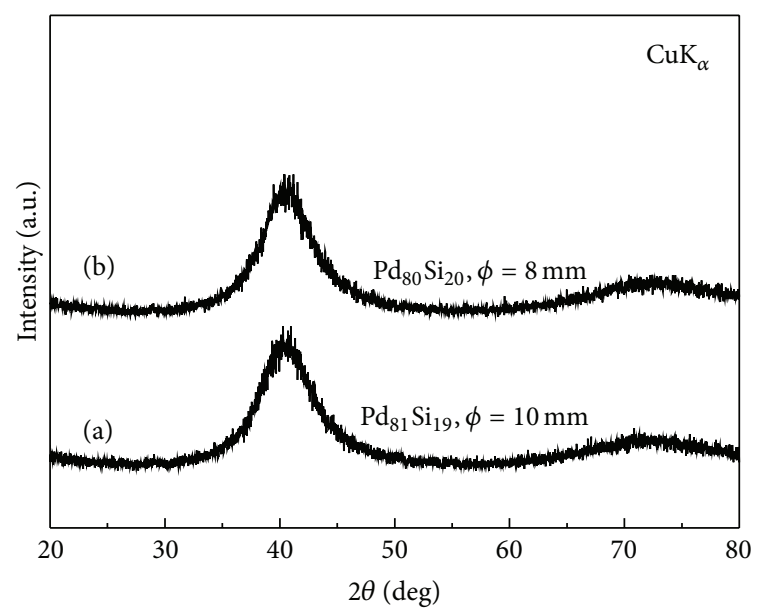

FIGURE 1: XRD patterns of Pd-Si alloys: (a) $\mathrm{Pd}_{81} \mathrm{Si}_{19} \mathrm{BMG}$ with a diameter up to $10 \mathrm{~mm}$ and (b) $\mathrm{Pd}_{80} \mathrm{Si}_{20} \mathrm{BMG}$ with a diameter up to $8 \mathrm{~mm}$.

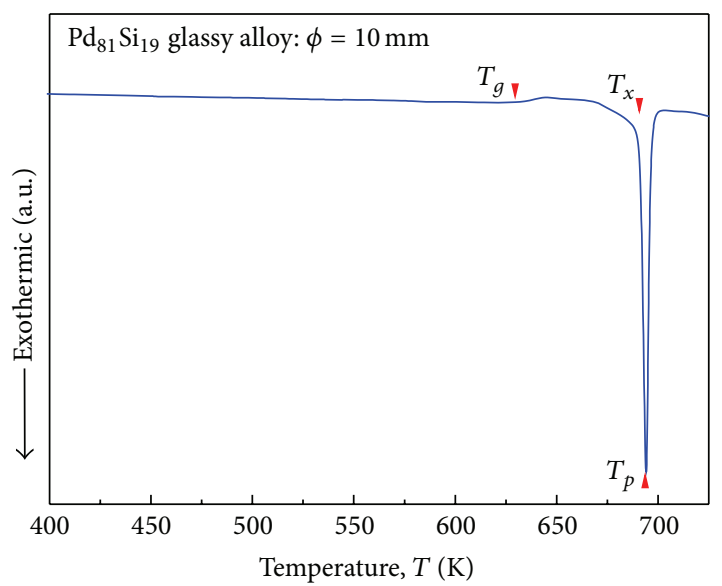

FIGURE 2: DSC curve of the $\mathrm{Pd}_{81} \mathrm{Si}_{19}$ BMG with a diameter of $10 \mathrm{~mm}$.

observed, indicating that glassy structure was formed in both samples.

Figure 2 presents the DSC curve of the $\mathrm{Pd}_{81} \mathrm{Si}_{19} \mathrm{BMG}$ with the near eutectic composition $\left(\mathrm{Pd}_{82.8} \mathrm{Si}_{17.2}\right)$. The glass transition temperature $\left(T_{q}\right)$, initial crystallization temperature $\left(T_{x}\right)$, and the peak crystallization temperature $\left(T_{p}\right)$ are determined to be $629 \mathrm{~K}, 691 \mathrm{~K}$, and $693 \mathrm{~K}$, respectively. Thus, the supercooled liquid region $\Delta T$ defined by the difference between $T_{g}$ and $T_{x}\left(\Delta T=T_{x}-T_{g}\right)$ can be obtained to be as large as $62 \mathrm{~K}$. Meanwhile, the DSC trace shows one distinct exothermic peak corresponding to the subsequent devitrification starting at $T_{x}$.

Figure 3 shows the structure of the $\mathrm{Pd}_{81} \mathrm{Si}_{19}$ BMG, which is typical for amorphous materials. No distinct evidence of crystalline diffraction spots or thin rings can be found in the selected-area electron diffraction (SAED) pattern shown in Figure 3(b). This further confirms the samples' amorphous structure. The high resolution transmission electron microscope (HRTEM) image evidences the structural inhomogeneity of the $\mathrm{Pd}_{81} \mathrm{Si}_{19} \mathrm{BMGs}$ as shown in Figure 3(c).

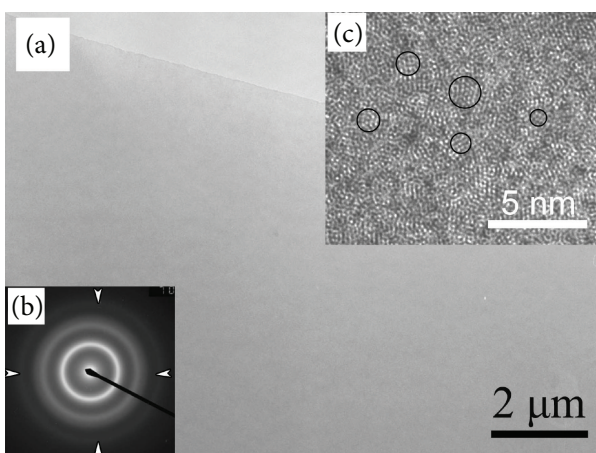

FIgURE 3: The microstructure of the $\mathrm{Pd}_{81} \mathrm{Si}_{19}$ BMG: (a) a bright field (BF) image, (b) a selected-area electron diffraction (SAED) pattern, and (c) a HRTEM image.

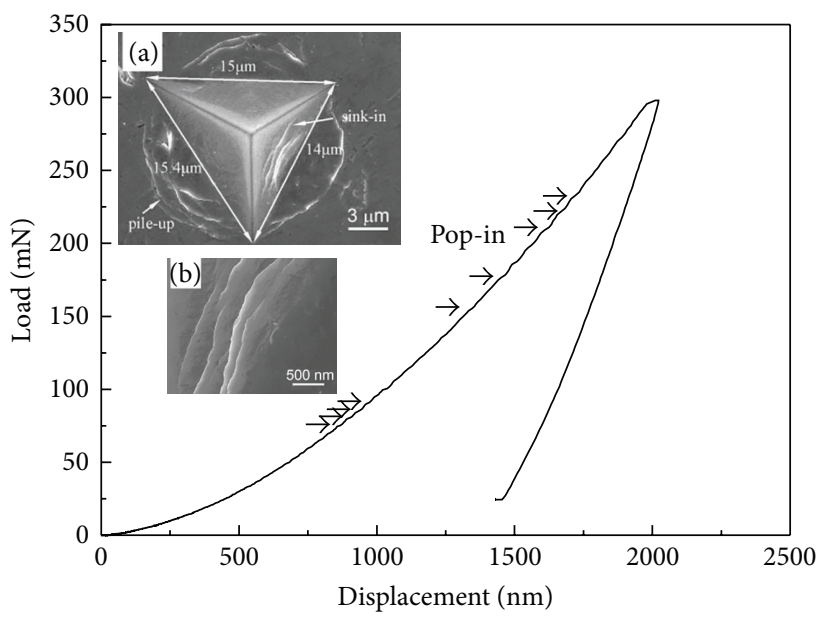

FIGURE 4: Nanoindentation load-displacement curve of the $\mathrm{Pd}_{81} \mathrm{Si}_{19}$ BMG with the inset: (a) SEM of indentation and (b) the enlargement of sinking-in.

For instance, the medium-range order (MRO) clusters less than $2 \mathrm{~nm}$ are frequently observed as indicated by the black circles, corresponding to the outer diffraction ring marked with white arrows shown in Figure 3(b).

Figure 4 shows the load-displacement $p$ - $h$ curve of the nanoindentation test for the $\mathrm{Pd}_{81} \mathrm{Si}_{19} \mathrm{BMG}$ at a strain rate of $0.05 \mathrm{~s}^{-1}$. A few small discrete pop-in events can be observed during the load process, indicating the operation of individual shear bands as reported in Zr-based, Ni-based, and other amorphous alloys [16-18]. The piling-up is observed as shown in the upper inset of Figure 4(a). Strikingly, the sinking-in phenomena also appear near the indenter, which are more pronounced for the strain-hardening or the nonstrain-hardening materials with a low value of $E / Y$ (where $E$ is elastic modulus and $Y$ is the yielding strength) shown in Figure 4(b) [19]. By using the ultrasonic measurements, the elastic moduli of Young's modulus, the shear modulus, and Poisson's ratio were obtained to be $94.53 \mathrm{GPa}, 33.43 \mathrm{GPa}$, and 0.414 , respectively. The yielding strength is about $1600 \mathrm{MPa}$ estimated from the compression test. Then the $E / Y$ can be calculated to be 59, a relatively low value compared 


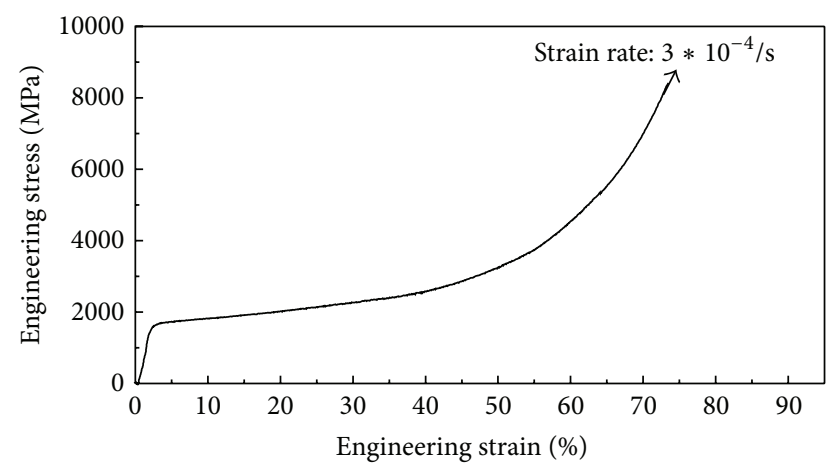

FiguRE 5: The compressive stress-strain curve of the $\mathrm{Pd}_{81} \mathrm{Si}_{19} \mathrm{BMG}$ deformed at room temperature.

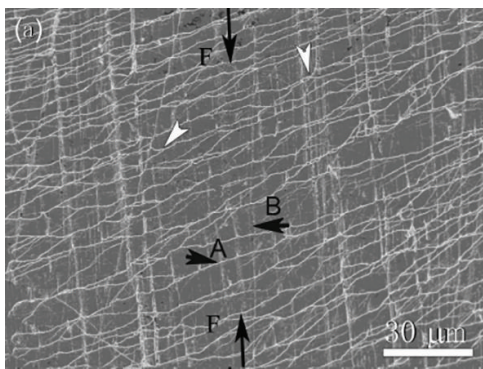

(a)

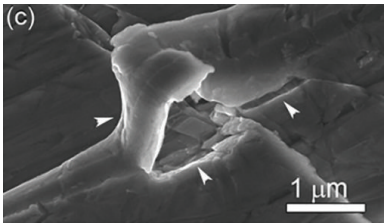

(c)

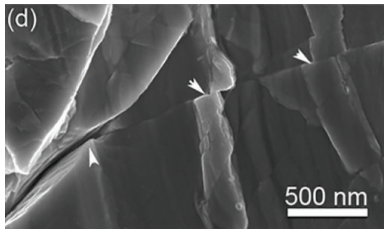

(d)

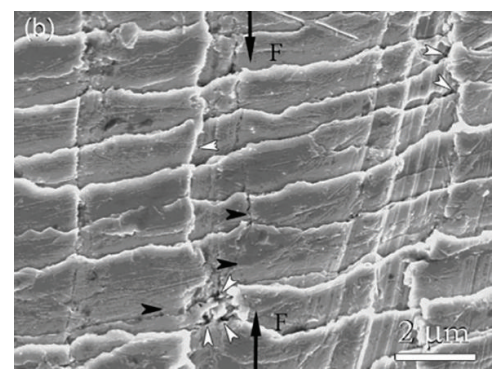

(b)

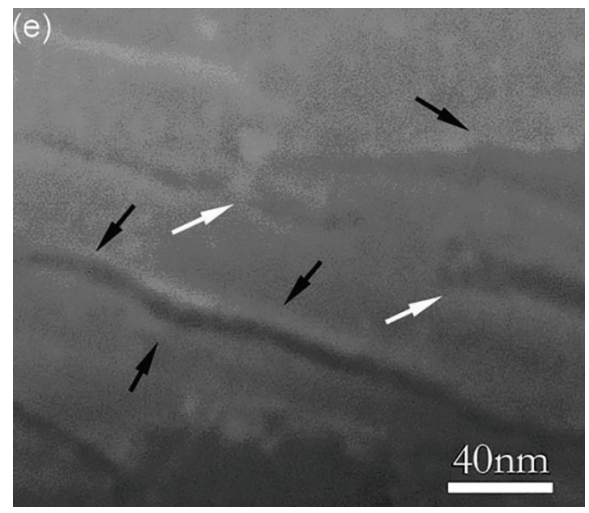

(e)

FIGURE 6: SEM side-view images of a deformed $\mathrm{Pd}_{81} \mathrm{Si}_{19}$ BMG specimen with a plastic engineering strain over $70 \%$ (a) $\times 2 \mathrm{~K}$, (b) $\times 20 \mathrm{~K}$, (c) $\times 100 \mathrm{~K},(\mathrm{~d}) \times 50 \mathrm{~K}$, and $(\mathrm{e}) \times 1000 \mathrm{~K}$.

with the corresponding crystalline materials. MGs usually show higher yielding strength and lower elastic modulus compared with their crystalline counterparts, leading to relatively low values of $E / Y$. MGs are thus expected to exhibit the sinking-in phenomena. However, most of the studies on the nanoindentation behaviors of MGs scarcely reported the presence of the sinking-in phenomena [16-18].

Figure 5 shows the compression curve of the $\mathrm{Pd}_{81} \mathrm{Si}_{19} \mathrm{BMG}$, showing a unique combination of a high strength of about $1600 \mathrm{MPa}$ and a large plasticity of over $70 \%$. The SEM side-view images of the compression specimen at a plastic engineering strain of $\sim 70 \%$ are shown in Figure 6(a). Different from most monolithic BMGs usually undergoing inhomogeneous plasticity and exhibiting poor ductility
$(<1 \%)$ at room temperature, quite uniform multiple shear bands are observed with an interspace ranging from 1 to $10 \mu \mathrm{m}$. Moreover, these shear bands branch and intersect as marked with white arrows. Meanwhile, many small steps of about $50-100 \mathrm{~nm}$ in size are formed along the shear bands (see Figures 6(a) and 6(b)). This indicates that the propagation of shear bands could be blocked, resulting in deflection, bifurcation, and branching. The macroscopic morphology of twisted shear bands is thus rough-and-tumble. In addition, the shear bands can be mainly divided into two types marked with black arrows shown in Figure 6(a): A type at an orientation of $\sim 70^{\circ}$ with respect to the compression direction and $\mathrm{B}$ type nearly parallel to the compression direction. Many A type shear 
bands are with angles of $35^{\circ} \sim 50^{\circ}$ to the loading axis. It is accepted that the macroscopic plasticity arises from the accumulation of local shear deformation originating from individual shear bands. So the total ductility increases with increasing the number of shear bands [20]. At a higher magnification image shown in Figure 6(b), voids marked by white arrows can be seen. Moreover, the initiation and propagation of the crack are also observed as marked by black arrows in Figure 6(b). Liu and coworkers reported that the formation of voids and cracks was induced by the tensile stress arising from the dilation and coalescence of the free volume [21]. The crack does not propagate along the shear bands but is hindered by the intersecting shear bands as shown in Figure 6(c). However, the intersecting shear bands pile up in Figure 6(d), exhibiting a softening behavior accompanied by microvoids formation.

The high resolution SEM image shows the morphology of microshear bands appearing between the main shear bands shown in Figure 6(e). Many kinks, deflection, and ledges marked by black arrows can be found along the shear bands. The heights of the ledges or kinks are about $10 \mathrm{~nm}$ or less. The interspace of these nanoscale shear bands is about 30$50 \mathrm{~nm}$. As indicated by the white arrows in Figure 6(e), the propagation of microshear bands can be locally arrested and the induced new shear bands can be observed ahead.

The nanoscale structural inhomogeneity in $\mathrm{Pd}_{81} \mathrm{Si}_{19}$ is suggested to account for its large plasticity. These MRO clusters could function as a reinforcing source to effectively hinder the further propagation of the matured shear bands and promote formation of new shear bands [22, 23]. Cao et al. also reported that strain hardening occurred in $\mathrm{Cu}-\mathrm{Zr}-\mathrm{Ti}$ metallic glass due to phase separation-induced inhomogeneity during the cold rolling process [24]. This also indicates that the structural inhomogeneity could help generate multiple shear bands contributing to large plasticity. Thus, designing a glass-forming alloy with nanoscale structural inhomogeneity could endow this alloy with a unique combination of high strength and large ductility.

\section{Conclusion}

$\mathrm{Pd}_{81} \mathrm{Si}_{19}$ binary BMGs were prepared in shape of spheres with diameters up to $10 \mathrm{~mm}$. The supercooled liquid region $\Delta T$ of the $\mathrm{Pd}_{91} \mathrm{Si}_{19}$ BMG is $62 \mathrm{~K}$, indicating that the $\mathrm{Pd}_{81} \mathrm{Si}_{19}$ alloys possess high thermal stability and good glass-forming ability. Both piling-up and sinking-in phenomena occurred during the nanoindentation tests, demonstrating a possible strain-hardening deformation mechanism. The uniaxial compression tests proved that $\mathrm{Pd}_{81} \mathrm{Si}_{19}$ glassy alloys exhibit the superductility with a compressive plastic strain over $70 \%$. Abundant shear bands were formed during deforming process, which is attributed to the intrinsically structural inhomogeneity.

\section{Conflict of Interests}

The authors declare that there is no conflict of interests regarding the publication of this paper.

\section{Acknowledgments}

This work is sponsored by Specialized Research Fund for the Doctoral Program of Higher Education, The National Science Foundation of China, and The Ministry of Science and Technology of China.

\section{References}

[1] W. Klement, R. H. Willens, and P. Duwez, "Non-crystalline structure in solidified gold-silicon alloys," Nature, vol. 187, no. 4740, pp. 869-870, 1960.

[2] A. L. Greer, "Metallic glasses," Science, vol. 267, no. 5206, pp. 1947-1953, 1995.

[3] W. L. Johnson, "Bulk glass-forming metallic alloys: science and technology," MRS Bulletin, vol. 24, no. 10, pp. 42-56, 1999.

[4] A. Inoue, "Stabilization of metallic supercooled liquid and bulk amorphous alloys," Acta Materialia, vol. 48, no. 1, pp. 279-306, 2000.

[5] W.-H. Wang, C. Dong, and C. H. Shek, "Bulk metallic glasses," Materials Science and Engineering R: Reports, vol. 44, no. 2-3, pp. 45-89, 2004.

[6] J. Schroers and W. L. Johnson, "Ductile bulk metallic glass," Physical Review Letters, vol. 93, no. 25, Article ID 255506, 2004.

[7] J. Das, M. B. Tang, K. B. Kim et al., "Work-hardenable' ductile bulk metallic glass," Physical Review Letters, vol. 94, no. 20, Article ID 205501, 2005.

[8] A. Inoue, W. Zhang, T. Tsurui, A. R. Yavari, and A. L. Greer, "Unusual room-temperature compressive plasticity in nanocrystal-toughened bulk copper-zirconium glass," Philosophical Magazine Letters, vol. 85, no. 5, pp. 221-237, 2005.

[9] K.-F. Yao, F. Ruan, Y. Q. Yang, and N. Chen, "Superductile bulk metallic glass," Applied Physics Letters, vol. 88, no. 12, Article ID 122106, 2006.

[10] Y. H. Liu, G. Wang, R. J. Wang, D. Q. Zhao, M. X. Pan, and W. H. Wang, "Super plastic bulk metallic glasses at room temperature," Science, vol. 315, no. 5817, pp. 1385-1388, 2007.

[11] D. Wang, Y. Li, B. B. Sun, M. L. Sui, K. Lu, and E. Ma, "Bulk metallic glass formation in the binary $\mathrm{Cu}-\mathrm{Zr}$ system," Applied Physics Letters, vol. 84, no. 20, pp. 4029-4031, 2004.

[12] D. Xu, B. Lohwongwatana, G. Duan, W. L. Johnson, and C. Garland, "Bulk metallic glass formation in binary $\mathrm{Cu}$-rich alloy series- $\mathrm{Cu}_{100-x} \mathrm{Zr}_{x}(x=34,36,38.2,40$ at.\%) and mechanical properties of bulk $\mathrm{Cu}_{64} \mathrm{Zr}_{36}$ glass," Acta Materialia, vol. 52, no. 9, pp. 2621-2624, 2004.

[13] L. Xia, D. Ding, S. T. Shan, and Y. D. Dong, "The glass forming ability of $\mathrm{Cu}$-rich $\mathrm{Cu}-\mathrm{Hf}$ binary alloys," Journal of Physics: Condensed Matter, vol. 18, no. 15, article 3543, 2006.

[14] L. Xia, W. H. Li, S. S. Fang, B. C. Wei, and Y. D. Dong, "Binary Ni-Nb bulk metallic glasses," Journal of Applied Physics, vol. 99, no. 2, Article ID 026103, 2006.

[15] N. Chen, Y. Li, and K.-F. Yao, "Thermal stability and fragility of Pd-Si binary bulk metallic glasses," Journal of Alloys and Compounds, vol. 504, supplement 1, pp. S211-S214, 2010.

[16] C. A. Schuh and T. G. Nieh, "A nanoindentation study of serrated flow in bulk metallic glasses," Acta Materialia, vol. 51, no. 1, pp. 87-99, 2003.

[17] J. G. Wang, B. W. Choi, T. G. Nieh, and C. T. Liu, "Crystallization and nanoindentation behavior of a bulk $\mathrm{Zr}-\mathrm{Al}-\mathrm{Ti}-\mathrm{Cu}-\mathrm{Ni}$ amorphous alloy," Journal of Materials Research, vol. 15, no. 3, pp. 798-807, 2000. 
[18] A. L. Greer, A. Castellero, S. V. Madge, I. T. Walker, and J. R. Wilde, "Nanoindentation studies of shear banding in fully amorphous and partially devitrified metallic alloys," Materials Science and Engineering A, vol. 375-377, no. 1-2, pp. 1182-1185, 2004.

[19] A. C. Fischer-Cripps, Nanoindentation, edited by F. F. Ling, Springer, New York, NY, USA, 2002.

[20] H. Bei, S. Xie, and E. P. George, "Softening caused by profuse shear banding in a bulk metallic glass," Physical Review Letters, vol. 96, no. 10, Article ID 105503, 2006.

[21] L. F. Liu, L. H. Dai, Y. L. Bai, B. C. Wei, and J. Eckert, "Behavior of multiple shear bands in Zr-based bulk metallic glass," Materials Chemistry and Physics, vol. 93, no. 1, pp. 174-177, 2005.

[22] K. L. Lee and H. W. Kui, "Phase separation in undercooled molten $\mathrm{Pd}_{80} \mathrm{Si}_{20}$ : part I," Journal of Materials Research, vol. 14, no. 9, pp. 3653-3662, 1999.

[23] S. Y. Hong, W. H. Guo, and H. W. Kui, "Metastable liquid miscibility gap in Pd-Si and its glass-forming ability: part III," Journal of Materials Research, vol. 14, no. 9, pp. 3668-3672, 1999.

[24] Q. Cao, J. Li, Y. Zhou, and J. Jiang, "Mechanically driven phase separation and corresponding microhardness change in $\mathrm{Cu}_{60} \mathrm{Zr}_{20} \mathrm{Ti}_{20}$ bulk metallic glass," Applied Physics Letters, vol. 86, no. 8, Article ID 081913, 2005. 

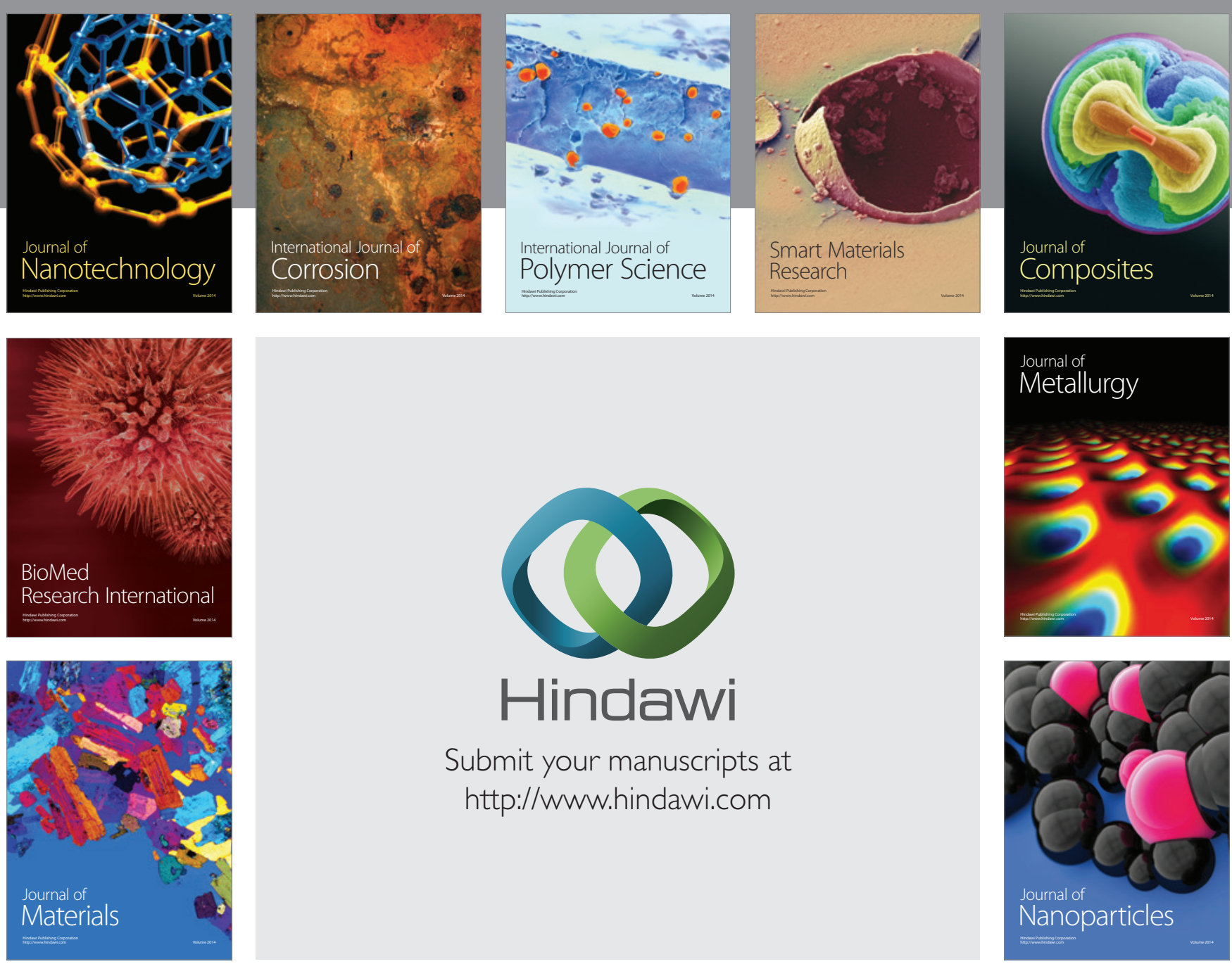

Submit your manuscripts at http://www.hindawi.com
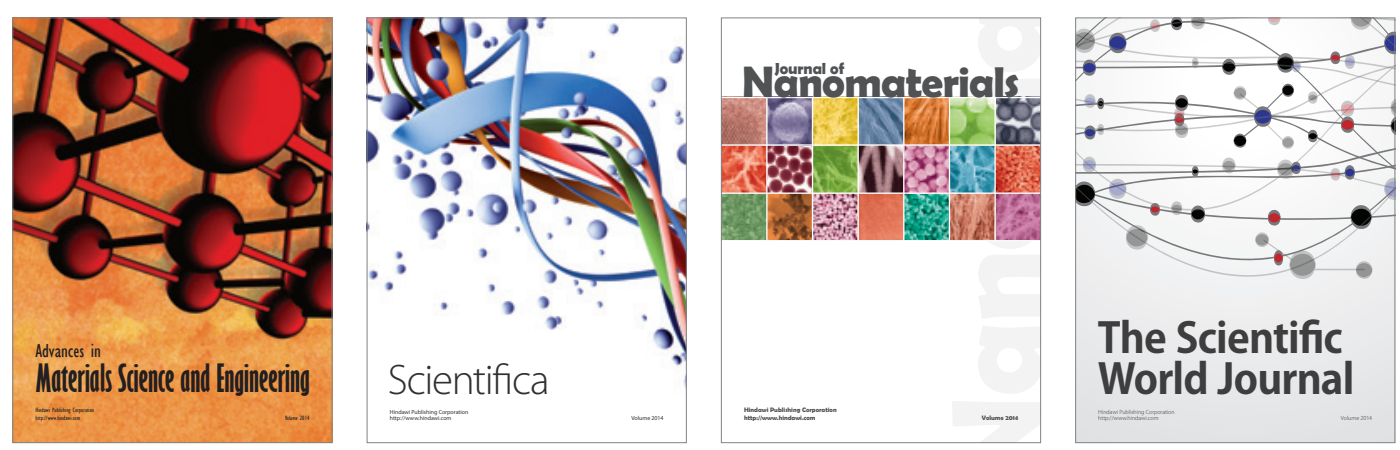

\section{The Scientific World Journal}
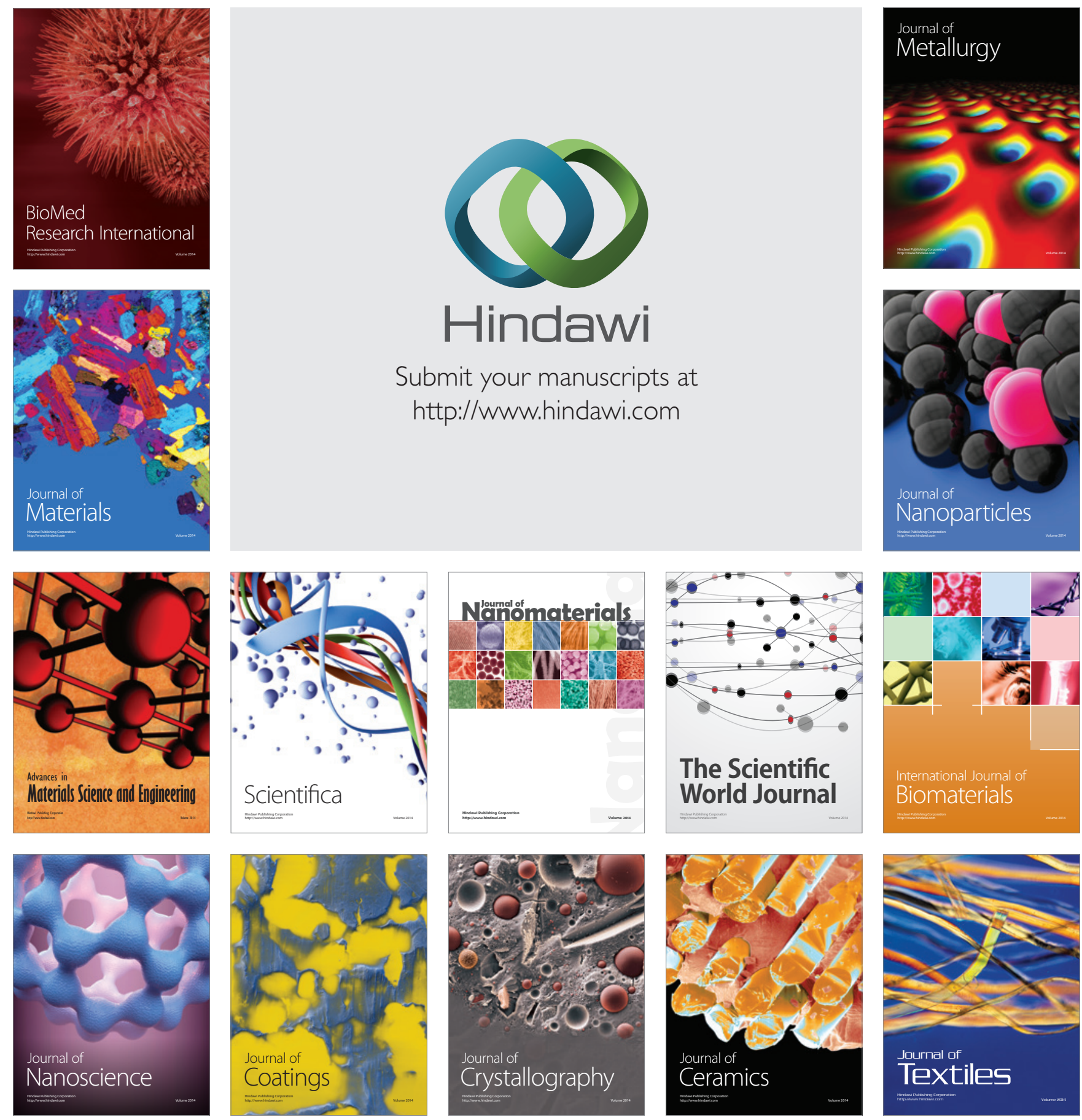